Impact of Iron Deficiency on Response to and Remodeling After Cardiac Resynchronization Therapy

Peer-reviewed author version

MARTENS, Pieter; VERBRUGGE, Frederik; NIJST, Petra; DUPONT, Matthias;

Tang, W. H. Wilson \& MULLENS, Wilfried (2017) Impact of Iron Deficiency on

Response to and Remodeling After Cardiac Resynchronization Therapy. In:

AMERICAN JOURNAL OF CARDIOLOGY, 119(1), p. 65-70.

DOI: 10.1016/j.amjcard.2016.09.017

Handle: http://hdl.handle.net/1942/23282 


\section{Impact of Iron Deficiency on Response to and Remodeling after Cardiac Resynchronization Therapy}

\section{Pieter Martens $M D^{1,2}$. Frederik Verbrugge $M D, P h D^{1}$. Petra Nijst $M D^{1,2}$. Matthias Dupont $M D^{1}$. W.H. Wilson Tang $M D^{3}$. Wilfried Mullens $M D, P h D^{1,4}$.}

1. Department of Cardiology, Ziekenhuis Oost-Limburg, Genk, Belgium

2. Doctoral School for Medicine and Life Sciences, Hasselt University, Diepenbeek, Belgium.

3. Department of Cardiovascular Medicine, Heart and Vascular Institute, Cleveland Clinic, Cleveland, $\mathrm{OH}$, United States of America.

4. Biomedical Research Institute, Faculty of Medicine and Life Sciences, Hasselt University, Diepenbeek, Belgium.

Grant Support: Pieter Martens is supported by a doctoral fellowship by the Research Foundation Flanders (FWO, grant-number: 1127917N). Pieter Martens, Petra Nijst, and Wilfried Mullens are researchers for the Limburg Clinical Research Program (LCRP) UHasselt-ZOL-Jessa, supported by the foundation Limburg Sterk Merk (LSM), Hasselt University, Ziekenhuis Oost-Limburg and Jessa Hospital.

Article short title: Impact of Iron Deficiency on Cardiac Resynchronization Therapy

\section{Corresponding author:}

Wilfried Mullens, M.D., Ph.D.

Department of Cardiology, Ziekenhuis Oost-Limburg

Schiepse Bos 6, 3600 Genk, BELGIUM

Tel: +3289327087 | Fax: +32 89327918 | E-mail: Wilfried.Mullens@zol.be 


\begin{abstract}
Iron deficiency is prevalent in heart failure with reduced ejection fraction (HFrEF) and relates to symptomatic status, readmission and all-cause mortality. The relationship between iron status and response to cardiac resynchronization therapy (CRT) remains insufficiently elucidated. This study assesses the impact of iron deficiency on clinical response and reverse cardiac remodeling as well as outcome after CRT. Baseline characteristics, change in New York Heart Association (NYHA) functional class, reverse cardiac remodeling on echocardiography, and clinical outcome (i.e. all-cause mortality and heart failure readmissions) were retrospectively evaluated in consecutive CRT patients who had full iron status and complete blood count available at implantation, implanted at a single tertiary care center with identical dedicated multi-disciplinary CRT follow-up between October, 2008 and August, 2015. A total of 541 patients were included with mean follow-up of $38 \pm 22$ months. Prevalence of iron deficiency was $56 \%$ at implantation. Patients with iron deficiency exhibited less symptomatic improvement 6 months after implantation ( $p$-value<0.001). In addition, both the decrease in left ventricular end-diastolic diameter ($3.1 \mathrm{~mm}$ versus $-6.2 \mathrm{~mm}$; $\mathrm{p}$-value $=0.011)$ and improvement in ejection fraction $(+11 \%$ versus $+15 \%, p$ value $=0.001$ ) were significantly lower in patients with iron deficiency. Iron deficiency was significantly associated with an increased risk for heart failure admission or all-cause mortality (adjusted $\mathrm{HR}, 95 \% \mathrm{Cl}=$ $1.718,1.178-2.506)$, irrespectively of the presence of anemia (Hemoglobin below $12 \mathrm{~g} / \mathrm{dl}$ in females and below $13 \mathrm{~g} / \mathrm{dl}$ in males). In conclusion, Iron deficiency is prevalent and affects both clinical response and reverse cardiac remodeling after CRT implantation. Moreover, it is a powerful predictor of adverse clinical outcomes in CRT.
\end{abstract}

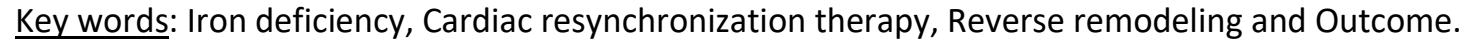




\section{Introduction}

Anemia is prevalent in patients with chronic heart failure and reduced ejection fraction (HFrEF) ${ }^{1}$. Substantial evidence suggests that it should be a target for post-implantation care following cardiac resynchronization therapy (CRT) ${ }^{2 ; 3}$. However, the pathophysiology of anemia is complex with hemodilution, occult blood loss, inflammation, renal disease and iron deficiency all playing a role ${ }^{4}$. Evidence linking anemia to CRT suggest that a high red cell distribution width and low mean corpuscular hemoglobin concentration relates to poor reverse remodeling, perhaps hinting to presence of iron deficiency ${ }^{5}$. Moreover, iron deficiency in HFrEF worsens prognosis and disability, irrespective of the presence of anemia ${ }^{6}$. Therefore, iron deficiency is increasingly being recognized as an import target in HFrEF amenable for intravenous supplementation even when anemia is not present $7 ; 8$. Given the pivotal role of iron in mitochondrial function, cardiac metabolism, oxygen transport, and cardiac remodeling, it seems feasible that iron deficiency might also affect the response and outcome after CRT ${ }^{9-11}$. However, there is a paucity of data with regards to iron status and the clinical response as well as potential impact on cardiac reverse remodeling following CRT. The objectives of this study are therefore: (1) to determine the prevalence of iron deficiency in CRT candidates; and (2) to assess the impact of iron deficiency at implantation on clinical response, reverse cardiac remodeling as well as outcome after CRT.

\section{Methods}

Consecutive HFrEF patients undergoing CRT implantation in a single tertiary care center (Ziekenhuis Oost-Limburg, Genk, Belgium) between October, 2008 and August, 2015, were evaluated. CRT indications were in compliance with the European Society of Cardiology guidelines ${ }^{12}$. For the current analysis, only patients with a full iron status (iron, ferritin and transferrin saturation) and complete blood count available at the time interval of 3 months before or after CRT implantation were included. Patients underwent a similar pre-specified thorough follow-up and dedicated post-implantation CRT optimization protocol, as published previously ${ }^{2 ; 13}$. Briefly, all patients received identical optimization of heart failure care including up-titration of neurohormonal blockers, as well as echocardiography-guided optimization of their device settings one day after implantation. This was repeated as indicated at each visit in a dedicated multidisciplinary CRT clinic ${ }^{2}$. Patients received a first follow-up appointment 6 weeks after 
implantation and a second follow-up at 6 months. The current study is in compliance with the Declaration of Helsinki. Given the retrospective nature of the study design, the need for written informed consent was waived by the local ethical committee.

Iron deficiency was defined according to established criteria ${ }^{7 ; 14}$. Both a ferritin level $<100 \mu \mathrm{g} / \mathrm{l}$ irrespectively of transferrin saturation (absolute iron deficiency) and a ferritin level 100-300 $\mathrm{\mu g} / \mathrm{l}$ with transferrin saturation $<20 \%$ (functional iron deficiency) were considered as iron deficiency. Anemia was defined as a hemoglobin level $<12 \mathrm{~g} / \mathrm{dl}$ in women and $<13 \mathrm{~g} / \mathrm{dl}$ in men according to the World Health Organization criteria. Patients undergoing transfusion or therapy with intravenous iron or oral iron during the index hospitalization and first 6 months of follow-up were excluded from the analysis. Comprehensive 2-dimensional echocardiography exams were performed (Philips Medical Systems, iE33w) at the time of device implantation and after 6 months. All reported measurements were averaged from 3 consecutive cycles (or 5 in case of atrial fibrillation) and assessed as recommended by the American Society of Echocardiography ${ }^{15}$. Left ventricular end-diastolic diameter (LVEDD) and end-systolic diameter (LVESD) were measured from the parasternal long axis view. Left ventricular ejection fraction (LVEF) was obtained using the modified Simpson's biplane method in the apical 2- and 4-chamber view.

Analysis of response to CRT was systematically assessed at 6 months after implantation. Symptomatic response was registered as a change in NYHA class between implantation and 6 months. Echocardiographic response was measured by the change in LVEF and LVEDD between implantation and 6 months. For the long term outcome analysis, the primary endpoint consisting of a combined endpoint of heart failure admission and all-cause mortality. Secondary endpoints consisted of each endpoint (heart failure hospitalization and all-cause mortality) separately. Heart failure admission was defined as an admission lasting $>24$ hours necessitating intravenous diuretics and presence of at least one sign and one symptom of congestion.

Normally distributed continuous variables were expressed as mean \pm standard deviation. Categorical data were expressed as numbers and $\%$ and compared with the Pearson $\chi^{2}$-test. Continuous variables were compared with the Student's $t$-test. Linear regression analysis was used to investigate the independent impact of iron deficiency on LVEF change and LVEDD change as a continuous outcome 
variable after adjusting for differences in baseline characteristics and known-predictors influencing reverse remodeling. The Kaplan-Meier method was used to construct survival curves, with the log-rank test used for comparison among groups. Unadjusted and adjusted hazard ratios were calculated by Cox regression analysis. Statistical significance was always set at a 2 -tailed probability level of $<0.05$. All statistics were performed using SPSS version 22 (IBM, Chicago, IL).

\section{Results}

From 686 consecutive CRT patients, a total of 547 had full iron status and complete blood count available at implantation and were considered for the current analysis. Mean time between baseline iron status and CRT-implantation was $3( \pm 56)$ days. Six patients were excluded from the analysis because they received iron or red blood cell transfusion during the index hospitalization or first six month follow-up, resulting in a final study population of 541 patients. Baseline characteristics are summarized in Table 1. Iron deficiency was present in 301 (56\%). Absolute versus functional iron deficiency was present in 162 patients (62\%) and 101 patients (38\%), respectively. Patients with iron deficiency were more likely to be women, diabetic, and more often received loop diuretics and anticoagulation therapy. The use of CRT devices with defibrillator was higher in patients without iron deficiency. When applying WHO-criteria for anemia, iron deficiency was not associated with a higher proportion of anemia, however on numerical basis the hemoglobin concentration was significantly lower (12.9 g/dl vs $13.6 \mathrm{~g} / \mathrm{dl}, \mathrm{p}=0.001)$.

Symptomatic improvement according to the change in NYHA functional class from implantation to 6 months of follow-up is depicted in Figure 1. Patients with iron deficiency had a significantly lower improvement in NYHA functional class ( $p$-value $<0.001)$ despite a similar distribution at baseline. No difference was seen between NYHA class changes between absolute and functional iron deficiency (supplementary table 1).

Reverse cardiac remodeling response according to iron status is presented in Table 2. Echocardiography with high image quality for consecutive measurements was available in 263 patients with iron deficiency (87\%) and 222 patients without (92\%). The proportion of patients dying in the iron deficient (4.7\%) and non-iron deficient (2.5\%) group before 6 months was equal $(p=0.252)$. Both groups 
demonstrated favorable reverse remodeling as indicated by a significant decrease in LVEDD and increase in LVEF (Table 2). However, the change in LVEDD and LVEF was significantly lower in patients with iron deficiency. After correcting for difference in baseline characteristics (table 1) and factors affecting reverse remodeling including female gender, Left bundle branch block, non-ischemic etiology and renal function, presence of iron deficiency at baseline remained independently predictive of diminished reverse remodeling (LVEF; $\beta$ expressed as $\%, \beta=-3.1 \pm 1.1, P=0.004$ and LVEDD; $\beta$ expressed as $m m, \beta=2.6 \pm 1.2$, $\mathrm{P}=0.036$ ). Moreover, no differences were observed between patients with absolute versus functional iron deficiency (supplementary table 2).

Six patients were lost to follow-up, with complete outcome data available for 535 patients (98\% of the total study population). A total 84 patients died and 53 patients were readmitted for heart failure during mean follow-up of $38 \pm 22$ months. In the primary endpoint analysis, 96 events ( 52 deaths and 44 heart failure readmissions) occurred in the iron deficiency group and 42 events (32 deaths and 10 heart failure readmissions) occurred in the group without iron deficiency (Figure $2 ; p<0.001$ ). Table 3 provides an overview of the unadjusted and adjusted cox regression analysis. Presence of iron deficiency resulted in a significant hazard ratio of $1.718(1.178-2.506)$ on the combined primary endpoint, mainly driven by heart failure hospitalization. Stratifying patients with iron deficiency according to the type of iron deficiency (absolute versus functional), resulted in similar event rate for the primary endpoint with a nonsignificant log-rank test at a p-value of 0.662 .

\section{Discussion}

This study provides important novel insights on the impact of iron deficiency on CRT: 1) iron deficiency is common at the time of CRT implantation; 2) patients with iron deficiency are less likely to improve their functional capacity after CRT; 3 ) iron deficiency in CRT patients is associated with less favorable reverse remodeling; 4) iron deficiency is associated with worse clinical outcome in CRT patients, irrespectively of the presence of anemia. These data suggest that screening for iron deficiency at the time of CRT implantation may help to identify a subgroup that could potentially benefit from intravenous iron therapy to improve CRT response. 
Iron deficiency is increasingly being recognized as an important comorbidity in HFrEF . Its reported prevalence ranges from $16 \%$ to $57 \%$ depending on the criteria used and population being studied ${ }^{16}$. Due to guideline-directed selection of CRT candidates, the current study included patients with symptomatic and advanced HFrEF ${ }^{12}$. It was therefore not surprising to find a high prevalence of iron deficiency in the current analysis. We also confirm previous findings that iron deficiency is often present even in the absence of anemia ${ }^{6}$.

In the setting of heart failure patients with a high prevalence of comorbidities, it is important to appreciate that symptomatic response not always neatly tracks echocardiographic response (reverse remodeling) or vice versa ${ }^{17}$. Results of the current study indicate that iron deficiency in CRT recipients influences the clinical response to CRT. From a patient perspective, this is an important observation, because even improvement of the most ingravescent symptoms of functional limitation will be regarded as success by the patient. Several well-conducted double-blind randomized controlled trials have demonstrated improvement in functional status of HFrEF patients with iron deficiency after administration of intravenous iron $7 ; 8$. Given the overlap between the inclusion criteria of these trials and the current analyzes, an extrapolation of treatment effect is probably safe to make. Moreover, little change was seen in the evolution of NYHA-class in the placebo groups of these trials, indicating that the current difference in NYHA class at 6 months is not due to a secular time effect of iron deficiency $7 ; 8$.

Our data suggests that HFrEF patients with iron deficiency at the time of CRT implantation exhibit a diminished capacity to reverse remodel. These results should be interpreted carefully because any meaningful conclusion on causality is hard to defer from a retrospective analysis. On the other hand, it is important to bear in mind that CRT is a clean non-pharmacological treatment for reverse remodeling that allows to assess the impact of a metabolic comorbidity on its reverse remodeling capacity. In that setting iron deficiency independently resulted after adjustment for baseline differences and factors influencing reverse remodeling in $-3.1 \pm 1.1 \%$ less improvement of LVEF and $2.6 \pm 1.2 \mathrm{~mm}$ more dilation of LVEDD. From a pathophysiologic perspective, two explanations might clarify this pivotal finding of reduced reverse remodeling. First, iron deficiency might be a barometer for disease severity, meaning that iron deficiency closely mirrors a more advanced disease state less capable of mounting reverse remodeling. Indeed, an 
important driver of cardiac remodeling is the pro-inflammatory state of heart failure (cytokine hypothesis), which also partially drives iron deficiency in heart failure. A recent study using circulating microRNA's illustrated that patients with a diminished level of micro-RNA30d exhibit a reduced ability to offset the detrimental effects of TNF- $\alpha$ leading to less reverse remodeling ${ }^{18}$. Thus, implying that higher pro-inflammatory states correlate with diminished reverse remodeling. However comparing functional versus absolute iron deficiency showed no difference between diminished reverse remodeling response. This might imply that iron deficiency itself and not the mechanism driving iron deficiency is responsible for the impaired reverse remodeling response. Secondly, we should attain the possibility that iron deficiency itself is not a barometer but mediator in the process of reverse remodeling. Rat data indicates that iron deficiency results in left ventricular dilatation, disorganized sarcomeres, mitochondrial swelling and increased mitochondrial emission of reactive oxygen species ${ }^{9-11 ; 19}$. CRT on the other hand results in numerous beneficial myocardial molecular changes, including sarcomere remodeling, redox-balance improvement, calcium cycling restoration, ion channel remodeling, improved sympatho-vagal balance and mitochondrial proteomic changes ${ }^{20-27}$. Given the overlapping themes between iron deficiency and cellular remodeling following CRT, it seems feasible that a normal (cardiac) iron balance is a prerequisite for optimal reverse remodeling. Therefore, it might be tempting to speculate whether administration of intravenous iron in iron deficient CRT candidates results in more favorable reverse remodeling. Two small randomized controlled trials suggested that intravenous iron results in an improvement of LVEF and LVEDD after 6 months in HFrEF patients (without CRT) ${ }^{28 ; 29}$. However larger prospective trials investigating intravenous iron in HFrEF have not systematically assessed the effect on reverse remodeling 7;8.

The current analysis also demonstrated that patients with baseline iron deficiency exhibit a higher rate of the primary end-point heart-failure admission or all-cause mortality. However this was mainly driven by the hospitalization for worsening heart failure and not mortality. Importantly, this detrimental effect of iron deficiency remained presence even after adjusting. The finding that patients with functional and absolute iron deficiency have equal readmission and mortality rates corroborates the hypothesis that iron deficiency itself is detrimental (and perhaps not the mechanism driving iron deficiency). 
Several limitations need to be addressed. First, this study was a retrospective analysis with classic limitations associated with this type of study design. Thereby making the study results mainly hypothesis generating. Nevertheless, all patients were included in a standardized optimization protocol and follow-up clinic, reflected by the low loss to follow-up (2\%). Secondly, only NYHA functional class was used as surrogate to assess functional improvement and this may be somewhat subjective. Indeed, iron deficiency has been linked to reduced cycloergospirometry or reduced 6 -minutes' walk test ${ }^{30}$. These functional investigations were not available. Third, only 547 patients of a total of 686 had an iron status and complete blood count available at implantation. Importantly, baseline characteristics of the studied population and patients excluded because of missing baseline iron measurements were similar (supplementary table 3). Fourth, it is important to emphasize that during the entire length of the study period, the scientific evidence for administration of intravenous iron in HFrEF was relatively low, which was reflected by the high prevalence of untreated iron deficiency and the low number of exclusions due to iron therapy in the analysis. Fifth, endpoints such as NYHA-class or reverse remodeling may be biased in a retrospective study, however investigators evaluating these outcome-parameters were blinded to the results of the iron or anemia status. Finally, we can only account for a potential impact of blood cell transfusion or iron therapy in the first 6 months, however both reverse remodeling and symptomatic improvement were assed in this time-frame. 


\section{References}

(1) Anand IS, Kuskowski MA, Rector TS. Anemia and change in hemoglobin over time related to mortality and morbidity in patients with chronic heart failure: results from Val-HeFT. Circulation 2005;112:11211127.

(2) Mullens W, Grimm RA, Verga T. Insights from a cardiac resynchronization optimization clinic as part of a heart failure disease management program. J Am Coll Cardiol 2009;53:765-773.

(3) Venkateswaran RV, Freeman C, Chatterjee N. Anemia and its association with clinical outcome in heart failure patients undergoing cardiac resynchronization therapy. J Interv Card Electrophysiol 2015;44:297-304.

(4) Kazory A, Ross EA. Anemia: the point of convergence or divergence for kidney disease and heart failure? J Am Coll Cardiol 2009;53:639-647.

(5) Rickard J, Kumbhani DJ, Gorodeski EZ. Elevated red cell distribution width is associated with impaired reverse ventricular remodeling and increased mortality in patients undergoing cardiac resynchronization therapy. Congest Heart Fail 2012;18:79-84.

(6) Jankowska EA, Rozentryt P, Witkowska A. Iron deficiency: an ominous sign in patients with systolic chronic heart failure. Eur Heart J 2010;31:1872-1880.

(7) Anker SD, Comin CJ, Filippatos G. Ferric carboxymaltose in patients with heart failure and iron deficiency. N Engl J Med 2009;361:2436-2448.

(8) Ponikowski P, van Veldhuisen DJ, Comin-Colet J. Beneficial effects of long-term intravenous iron therapy with ferric carboxymaltose in patients with symptomatic heart failure and iron deficiencydagger. Eur Heart J 2015;36:657-668.

(9) Dong F, Zhang X, Culver B, Chew HG, Jr., Kelley RO, Ren J. Dietary iron deficiency induces ventricular dilation, mitochondrial ultrastructural aberrations and cytochrome c release: involvement of nitric oxide synthase and protein tyrosine nitration. Clin Sci (Lond) 2005;109:277-286. 
(10) Naito Y, Tsujino T, Matsumoto M, Sakoda T, Ohyanagi M, Masuyama T. Adaptive response of the heart to long-term anemia induced by iron deficiency. Am J Physiol Heart Circ Physiol 2009;296:H585-H593.

(11) Turner LR, Premo DA, Gibbs BJ. Adaptations to iron deficiency: cardiac functional responsiveness to norepinephrine, arterial remodeling, and the effect of beta-blockade on cardiac hypertrophy. BMC Physiol 2002;2:1.

(12) McMurray JJ, Adamopoulos S, Anker SD. ESC guidelines for the diagnosis and treatment of acute and chronic heart failure 2012: The Task Force for the Diagnosis and Treatment of Acute and Chronic Heart Failure 2012 of the European Society of Cardiology. Developed in collaboration with the Heart Failure Association (HFA) of the ESC. Eur J Heart Fail 2012;14:803-869.

(13) Mullens W, Kepa J, De VP. Importance of adjunctive heart failure optimization immediately after implantation to improve long-term outcomes with cardiac resynchronization therapy. Am J Cardiol 2011;108:409-415.

(14) Okonko DO, Grzeslo A, Witkowski T. Effect of intravenous iron sucrose on exercise tolerance in anemic and nonanemic patients with symptomatic chronic heart failure and iron deficiency FERRIC-HF: a randomized, controlled, observer-blinded trial. J Am Coll Cardiol 2008;51:103-112.

(15) Schiller NB, Shah PM, Crawford M. Recommendations for quantitation of the left ventricle by twodimensional echocardiography. American Society of Echocardiography Committee on Standards, Subcommittee on Quantitation of Two-Dimensional Echocardiograms. I Am Soc Echocardiogr $1989 ; 2: 358-367$.

(16) Jankowska EA, von HS, Anker SD, Macdougall IC, Ponikowski P. Iron deficiency and heart failure: diagnostic dilemmas and therapeutic perspectives. Eur Heart J 2013;34:816-829.

(17) Verbrugge FH, Dupont M, Rivero-Ayerza M. Comorbidity significantly affects clinical outcome after cardiac resynchronization therapy regardless of ventricular remodeling. J Card Fail 2012;18:845-853. 
(18) Melman YF, Shah R, Danielson K. Circulating MicroRNA-30d Is Associated With Response to Cardiac Resynchronization Therapy in Heart Failure and Regulates Cardiomyocyte Apoptosis: A Translational Pilot Study. Circulation 2015;131:2202-2216.

(19) Munzel T, Gori T, Keaney JF, Jr., Maack C, Daiber A. Pathophysiological role of oxidative stress in systolic and diastolic heart failure and its therapeutic implications. Eur Heart J 2015;36:2555-2564.

(20) DeMazumder D, Kass DA, O'Rourke B, Tomaselli GF. Cardiac resynchronization therapy restores sympathovagal balance in the failing heart by differential remodeling of cholinergic signaling. Circ Res 2015;116:1691-1699.

(21) Lichter JG, Carruth E, Mitchell C. Remodeling of the sarcomeric cytoskeleton in cardiac ventricular myocytes during heart failure and after cardiac resynchronization therapy. J Mol Cell Cardiol 2014;72:186-195.

(22) Kirk JA, Holewinski RJ, Kooij V . Cardiac resynchronization sensitizes the sarcomere to calcium by reactivating GSK-3beta. J Clin Invest 2014;124:129-138.

(23) Aiba T, Barth AS, Hesketh GG. Cardiac resynchronization therapy improves altered Na channel gating in canine model of dyssynchronous heart failure. Circ Arrhythm Electrophysiol 2013;6:546-554.

(24) Chakir K, Depry C, Dimaano VL. Galphas-biased beta2-adrenergic receptor signaling from restoring synchronous contraction in the failing heart. Sci Trans/ Med 2011;3:100ra88.

(25) Wang SB, Foster DB, Rucker J, O'Rourke B, Kass DA, Van Eyk JE. Redox regulation of mitochondrial ATP synthase: implications for cardiac resynchronization therapy. Circ Res 2011;109:750-757.

(26) Agnetti G, Kaludercic N, Kane LA. Modulation of mitochondrial proteome and improved mitochondrial function by biventricular pacing of dyssynchronous failing hearts. Circ Cardiovasc Genet 2010;3:78-87.

(27) Vanderheyden M, Mullens W, Delrue L. Myocardial gene expression in heart failure patients treated with cardiac resynchronization therapy responders versus nonresponders. J Am Coll Cardiol 2008;51:129-136. 
(28) Usmanov RI, Zueva EB, Silverberg DS, Shaked M. Intravenous iron without erythropoietin for the treatment of iron deficiency anemia in patients with moderate to severe congestive heart failure and chronic kidney insufficiency. J Nephrol 2008;21:236-242.

(29) Toblli JE, Lombrana A, Duarte P, Di GF. Intravenous iron reduces NT-pro-brain natriuretic peptide in anemic patients with chronic heart failure and renal insufficiency. J Am Coll Cardiol 2007;50:16571665.

(30) Jankowska EA, Rozentryt P, Witkowska A . Iron deficiency predicts impaired exercise capacity in patients with systolic chronic heart failure. J Card Fail 2011;17:899-906.

Figure legends

Figure 1. Symptomatic response at 6 month follow-up.

Figure 2: Long term outcome according to the presence of iron deficiency at implantation 